\title{
Riboswitches as Potential Targets for Aminoglycosides Compared with rRNA Molecules: In Silico Study
}

\author{
Elnaz Mehdizadeh Aghdam ${ }^{1,2}$, Mohammad Esmaeil Hejazi ${ }^{3}$, Mohammad Saeid Hejazi ${ }^{*}$ and Abolfazl Barzegar $^{4}$ \\ ${ }^{1}$ Department of Pharmaceutical Biotechnology, Faculty of Pharmacy, Tabriz University of Medical Sciences, Tabriz, Iran \\ ${ }^{2}$ Student Research Committee, Tabriz University of Medical Sciences, Tabriz, Iran \\ ${ }^{3}$ Faculty of Medicine, Tabriz University of Medical Sciences, Tabriz, Iran. \\ ${ }^{4}$ Research Institute for Fundamental Sciences (RIFS), University of Tabriz, Tabriz, Iran
}

\begin{abstract}
Riboswitches are cis acting riboregulaters in non-coding region of the mRNAs. Their possible contribution in antibiotic targeting especially for FMN, TPP and lysine riboswitches in bacteria has been revealed since a decade ago. Regarding some studies on the possibility of the interaction between aminoglycosides and the artificial riboswitches, in this study we attempted to evaluate the binding potential of different types of aminoglycosides including gentamicin, amikacin, kanamycin, neomycin, tobramaycin, sisomicin and paromomycin with various classes of riboswitches using computational methods. Applying Auto Dock vina, it was shown that the binding energy of each kind of riboswitches with different types of aminoglycosides (riboswitches/aminoglycosides) is almost similar or sometimes more than the binding energy of the aminoglycoside with the corresponding binding cage of "16S rRNA A site" (16S rRNA A site/aminoglycosides) as aminoglycosides' target site. The affinity between riboswitches and aminoglycosides is almost the same or higher than the affinity of riboswitches/natural ligands. In this study ampicillin was used as the negative control antibiotic and 5S rRNA was employed as the negative control RNA. Results showed that the binding energies of riboswitches/ampicillin and $5 \mathrm{~S}$ rRNA/aminoglycosides are usually lower than the energy of riboswitches/aminoglycosides. Accordingly, lysine, glycine and SAM-I riboswitches were recognized as the best RNA targets for all of the aminoglycosides because of their higher binding energy. In the next step, docking results were further validated by rDock program. Furthermore, it was shown that hydrogen binding makes a key role in the binding energy between aminoglycosides and riboswitches. Moreover, MD simulation studies on lysine riboswitch/paromomycin complex confirmed the stability of the docked structure in the solvent containing magnesium and chloride ions.
\end{abstract}

Keywords: Riboswitches; Aminoglycosides; Docking; Binding energy; rRNA

\section{Introduction}

Riboswitches as non-coding sequences usually located in 5'UTR of mRNAs, are involved in gene regulation through binding to various small molecules without protein interpretation. Ligand binding to the conserved structure of riboswitches causes the folding shift of RNA molecule and halting of transcription and/or translation of downstream related genes [1]. Their unique characteristics on specific and selective binding to various molecules make them interesting biological devices. Since early 2000s, when the riboswitches were primarily introduced [2], the possibility of their application as antibiotic targets has been raised dramatically. At the beginning, the riboswitch-targeting mechanism of four well-known antibiotics including roseoflavin [3,4], pyrithiamine [5], L-Aminoethylcysteine (AEC) and DL-4-oxalysine [6] were approved as ligands for FMN, TPP and lysine riboswitches. Afterwards, some efforts have been taken to find drug-like compounds through drug discovery methods in order to achieve some reliable effective antibacterials.

The aminoglycoside antibiotics are important therapeutic compounds in the treatment of severe bacterial infections. They exert their effects via binding to A site of $16 \mathrm{~S}$ rRNA in 30 S ribosomal subunit and cause interference in mRNA translation [7-9]. The presence of the cationic amine groups of the aminoglycosides causes binding to the negatively charged pockets in folded RNA [10]. However, in addition to "16S rRNA A site", it has been shown that aminoglycoside neomycin could bind to several other RNAs including the TransActivating Response (TAR) [11], Rev response element (RRE) RNAs of HIV-1[12] and catalytic RNAs, such as group I introns, RNase P, and the hammerhead and hepatitis delta virus ribozymes [13-16]. It is established that conformational changes in the RNA molecules can be occurred by drug binding at such sites $[17,18]$. The binding capability of the aminoglycosides to folded RNA structures has been applied to produce RNA aptamers [19]. In addition, designing artificial riboswitches for aminoglycosides have been conducted in the past decade [20-23]. For instance, a neomycin B binding artificial riboswitch was designed [20] which have later been studied structurally for the binding of ribostamycin and tobramycin [22]. Moreover, an in vitro interaction evaluation has been shown that some aminoglycosides inhibit the glms riboswitch [24].

According to the literature, there have been some reports about the structural similarity between riboswitches and rRNAs which raises the

*Corresponding authors: Mohammad Saeid Hejazi, Department of Pharmaceutical Biotechnology, Faculty of Pharmacy, Tabriz University of Medical Sciences, Tabriz, Iran, Tel: +98 41 33372256; Fax: +98 41 33344798; E-mail: saeidhejazi@tbzmed.ac.ir; msaeidhejazi@yahoo.com

Abolfazl Barzegar, Research Institute for Fundamental Sciences (RIFS), University of Tabriz, Tabriz, Iran, Tel: +98 4113294114; Fax: +98 411 294113; E-mail: barzegar@tabrizu.ac.ir

Received July 29, 2014; Accepted November 21, 2014; Published November 28,2014

Citation: Mehdizadeh Aghdam E, Esmaeil Hejazi M, Hejazi MS, Barzegar A (2014) Riboswitches as Potential Targets for Aminoglycosides Compared with rRNA Molecules: In Silico Study. J Microb Biochem Technol S9: 002. doi:10.4172/19485948.S9-002

Copyright: (c) 2014 Mehdizadeh Aghdam E, et al. This is an open-access article distributed under the terms of the Creative Commons Attribution License, which permits unrestricted use, distribution, and reproduction in any medium, provided the original author and source are credited 
possibility of functional connection between these two types of RNA molecules. In 2007 some structurally similar motifs to rRNA structures have been detected in riboswitches [25]. In 2008, an artificial riboswitch for neomycin B (an aminoglycoside antibiotic), was engineered [20] which partially resembles the ribosomal A site, the natural target for aminoglycoside antibiotics [26]. A comprehensive study on this similarity was carried out by our group [27]. Regarding the structural similarity between rRNAs and riboswitches, the possibility of binding of paromomycin, as a representative of aminoglycosides, to different types of riboswitches was verified [27]. In this study we are aimed to evaluate and validate the binding potential of 7 aminoglycosides including paromomycin, gentamicin, amikacin, kanamycin, neomycin, tobramaycin, sisomicin against 9 types of riboswitches through computational methods.

\section{Methods}

\section{Molecular docking}

Molecular docking is an important tool in structural biology and computational drug design. It is commonly used to calculate the binding modes of ligands and drug candidates to their protein/nucleic acid targets to predict the affinity and activity of the small molecule drugs [28]. Consequently, there is a wide range of uses and applications for molecular docking, including drug discovery and affinity prediction [29]. The most cited docking tool, namely Auto Dock [30] was used to predict the binding orientation of aminoglycosides to 9 types of riboswitches. The last version of Auto Dock namely Auto Dock Vina significantly enhances the average accuracy and speed of the binding mode predictions compared to Auto Dock 4 for molecular docking [31].

\section{Preparation of Macromolecules for AutoDock Vina}

Nine riboswitch classes which have not only the most representatives in microorganisms [32], but also have available PDB structures, were selected. Their PDB codes which represent preferably unbound state of riboswitches were extracted first from Rfam (http://rfam.sanger.ac.uk/) and then PDB (http://www.rcsb.org/pdb/home/home.do) databases. These riboswitch classes included TPP (PDB code: 2gdi), FMN (PDB code: 2 yie), SAM-I (PDB code: 3iqn), lysine (PDB code: $3 \mathrm{~d} 0 \mathrm{x}$ ), glycine (PDB code: 3ox0), purine (PDB code: 4fe5), c-di-GMP-II (PDB code: 3q3z), preQ1 (PDB code: 3fu2), THF (PDB code: 3suy) riboswitches. In addition, the PDB structures of " $16 \mathrm{~S}$ rRNA A site" were obtained from $1 \mathrm{j} 7 \mathrm{t}$, 2et3, 2g5q, 2esi, 2ets, 1lc4, 4f8u codes for paromomycin, gentamicin, amikacin, kanamycin, neomycin, tobramaycin and sisomicin, respectively. 5S Ribosomal RNA structure was extracted from 1c2x PDB code and was used as a negative RNA control. Water and ligand molecules were eliminated by the software program Viewer Pro Version 5.0. In addition, non-polar hydrogens and Gasteiger charges were added during the preparation of the macromolecule input file using the Auto Dock Tools package 1.5.6 [30].

\section{Preparation of the ligand for Auto Dock Vina}

The structures of seven aminoglycosides including paromomycin, gentamicin, amikacin, kanamycin, neomycin, tobramaycin and sisomicin were obtained through splitting of PDB codes of "16S rRNA A site" including $1 \mathrm{j} 7 \mathrm{t}, 2 \mathrm{et} 3,2 \mathrm{~g} 5 \mathrm{q}, 2 \mathrm{esi}, 2 \mathrm{ets}, 1 \mathrm{lc} 4,4 \mathrm{f} 8 \mathrm{u}$, respectively, using Viewer Pro Version 5. In addition, the specific ligand of each riboswitch was split from the complex structure of the riboswitch from PDB codes of 3d0u, 3owi, 2yie, 3iqr, 3suh, 3fu2, 4fe5, 3q3z, 2gdi for lysine, glycine, FMN, SAM, THF, preQ, purine, c-di-GMP-II and TPP molecules, respectively. Besides, the structure of ampicillin as a negative control antibiotic was acquired from 4kr4 PDB code. All rotatable bonds within the ligands were allowed to rotate freely and Gasteiger charges were added to the obtained structure of the ligand using the Auto Dock Tools package 1.5.6.

\section{Docking procedure}

All dockings were performed using Auto Dock Vina 1.1.2 [31] which is a new generation of docking software from the Molecular Graphics Lab (http://vina.scripps.edu). The grid box of each riboswitch was set according to the similar part of the riboswitches with "16S rRNA A site" based on our previous study [27]. In addition, the grid boxes for various A sites were obtained based on the binding site of the aminoglycosides. The number of 20 modes was set for each docking run. Other parameters were kept to their default values. First, docking was carried out for every aminoglycoside and riboswitches. The pdbqt file of each docking procedure was created and the best binding energy was acquired. The binding energies of the aminoglycosides with related "16S rRNA A sites" and also the binding energies of the riboswitches with their own specific ligands were considered as positive controls. Besides, the interaction between riboswitches and ampicillin as well as the interaction of 5S rRNA with aminoglycosides were considered as negative controls. The interaction features of selected conformations were analyzed using AutoDockTools package 1.5.6.

\section{Docking validation}

rDock program is a fast and versatile docking tool for docking small molecules against for nucleic acids [33]. Docked conformations of paromomycin and gentamicin (as sample aminoglycosides) with highest binding energy in Auto Dock Vina were selected to be validated and rescored via rDock.

\section{Docking preparation for rDock}

At first the system definition parameter file was developed to define the receptor file, ligand and scoring functions. Cavity mapping was carried out based on "Reference ligand method" and the radius of cavity mapping region, radius of small probe, minimum cavity volume to accept, 'maximum number of cavities to accept, receptor atom radius increment for excluded volume and grid resolution for mapping, were considered $4.0 \AA$, $1.0 \AA, 100 \AA 3,10,0.0 \AA$ and $0.5 \AA$, respectively. Receptor and ligand files were prepared in Mol2 and SD formats, respectively, using Open Babel 2.3.2 program. Finally cavity mapping was performed using rbcavity (one of the executable programs of rDock). Besides, where needed rbrmsd was used to calculate the RMSD between pose predictions and the PDB structure.

\section{Docking processing for rDock}

Having done the cavity mapping, docking process was performed using rbdock with definition of input files including ligand, system definition parameter file, docking protocol file and output SD format file. The file dock_solv.prm was considered as the docking protocol file based on SF5 scoring function which is compatible with nucleic acids according to the reference guide [33].

\section{Post-processing and analysis of results}

In order to sort out the docking conformations based on the total score, sdsort program was utilized. The output SD file of rbdock was considered as input file for sdsort. Afterwards, the summary of scores (total, inter, intra and $\mathrm{vdw}$ ) was obtained through sdreport program. According to the program guide, total score is a weighed sum of intermolecular, ligand intramolecular, site intramolecular and external 
restraint terms. Inter score is the most important term as it represents the receptor-ligand interaction score. Intra score shows the energy difference between the ligand and the input ligand conformation.

\section{Molecular dynamics simulation}

In order to confirm the data from docking results we performed additional computational molecular dynamics (MD) simulation approach via GROMACS program [34]. For this aim, the complex (lysine riboswitch/paromomycin) was placed in a cubic box center of $12.164 \mathrm{~nm}, 12.164 \mathrm{~nm}, 12.164 \mathrm{~nm}$ with period boundary condition and solvated by TIP3P water molecules [35]. $\mathrm{Mg}^{2+}$ and $\mathrm{Cl}^{-}$counterions were added to maintain overall system electroneutrality. The simulation was performed with GROMACS 5.0 suite program using CHARMM27 force field [36]. The Berendsen temperature coupling was used to keep the system at $300 \mathrm{~K}$, and the constant of coupling was $0.1 \mathrm{ps}$. The Particle Mesh Ewald (PME) algorithm was applied to calculate longrange electrostatics interactions with a cutoff of $0.9 \mathrm{~nm}$ and a cutoff of $1.4 \mathrm{~nm}$ was set for Van der Waals interactions. The Verlet leapfrog integrator with an integration time step of $2 \mathrm{fs}$ was used and LINCS algorithm was employed to keep all bonds involving hydrogen atoms rigid.

At first, the system was subjected to 500000 steps of steep descent energy minimization. Then, position restrained molecular dynamics was subsequently carried out for 200 ps. Finally, MD simulation was run for 2000 ps to the whole system.

\section{Results}

\section{Docking of riboswitches with various aminoglycosides}

Auto Dock Vina [37] is an open-source program for molecular docking. In comparison to Auto Dock 4, Auto Dock Vina significantly enhances the average accuracy of the binding predictions. Docking was performed on 9 types of riboswitches (lysine, glycine, purine, FMN, SAM, TPP, PreQ, c-di-GMP-II, THF) as receptors interacting with 7 types of aminoglycosides (paromomycin, gentamicin, amikacin, kanamycin, neomycin, tobramaycin, sisomicin) as ligands using Auto Dock Vina. The rRNA molecule was used as a control for all of 7 types of aminoglycosides because of having "16S rRNA A site" as a known binding site for aminoglycosides. Therefore, the docked results for riboswitches/aminoglycosides were compared with the binding energy of docked antibiotics with " $16 \mathrm{~S}$ rRNA A site". Moreover, the corresponding natural metabolites of riboswitches were docked and used as a positive control (named own ligand) to assay antibiotic interactions with riboswitches (Figures 1-7). The RMSD calculated by $\mathrm{rDock}$ between predicted poses of natural ligands and their PDB structure were almost $1 \sim 2 \mathrm{~A}^{\circ}$. The interactions of some complexes including the $5 \mathrm{~S}$ rRNA/ aminoglycosides and riboswitches/ ampicillin were considered as negative controls. The binding energy of such complexes was almost 3-4 kcal/mol (Figure 1-7).

As seen in Figure 1, the binding energy of all of the riboswitches/ paromomycin is approximately close to the binding energy of the interaction between rRNA A site/paromomycin. It was revealed that the binding energy of every kind of riboswitches/paromomycin is more (negatively) than the binding energy of riboswitches/natural ligands except for c-di-GMP-II riboswitch. In addition, the affinity of aminoglycosides/riboswitches is almost 2 -fold of the affinity of riboswitches/ampicillin and 5S rRNA/paromomycin (Figure 1-8). Besides, the binding energies of the riboswitches/aminoglycosides are comparable to those of riboswitches/natural ligands. Even the binding energies of the glycine, lysine and purine riboswitches/aminoglycosides are higher than those of riboswitches/natural ligands. In addition,

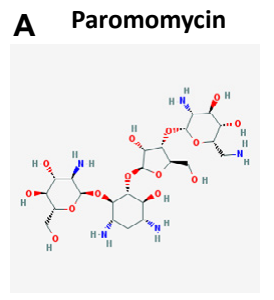

B

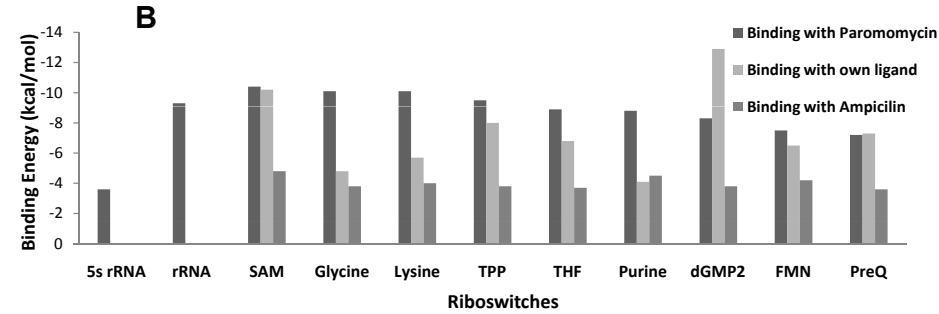

C
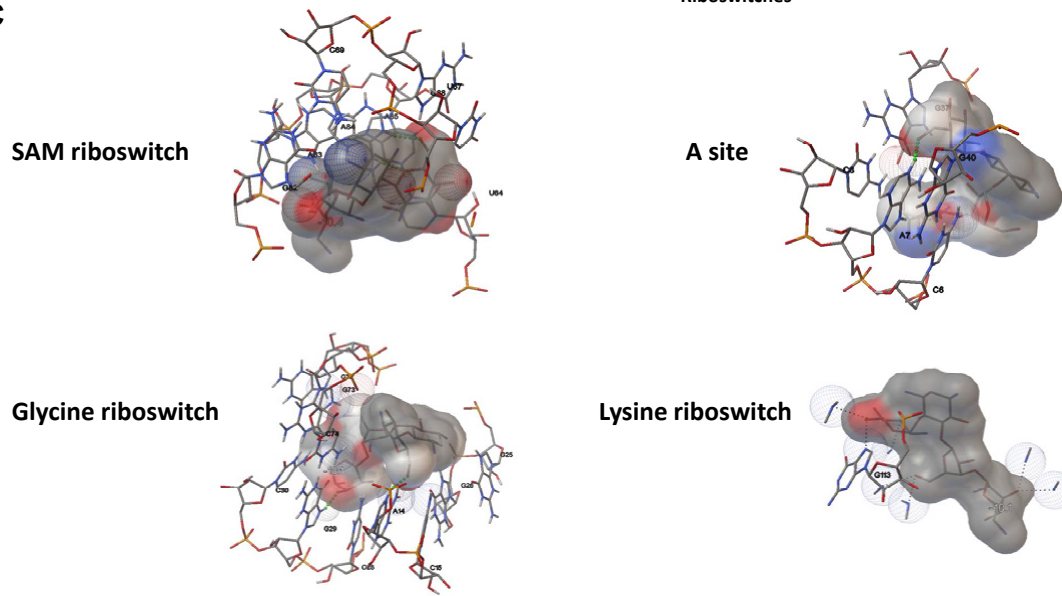

Lysine riboswitch

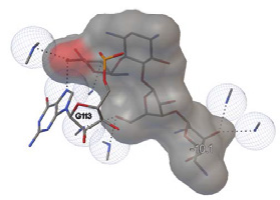

Figure 1: (A) the chemical structure of paromomycin. (B) Dark black columns demonstrate the binding energies of paromomycin with different riboswitches. Dark grey columns show the binding energy of each riboswitch with its own natural ligands. Light grey columns show the binding energy of each riboswitch with ampicillin (C) The interaction between the riboswitches and paromomycin. Hydrogen bindings were shown as green dot lines. 
A

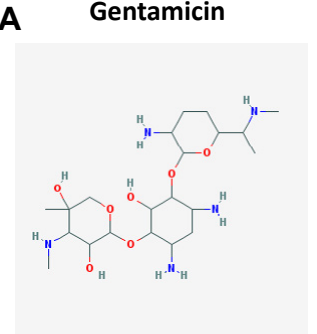

C

SAM riboswitch
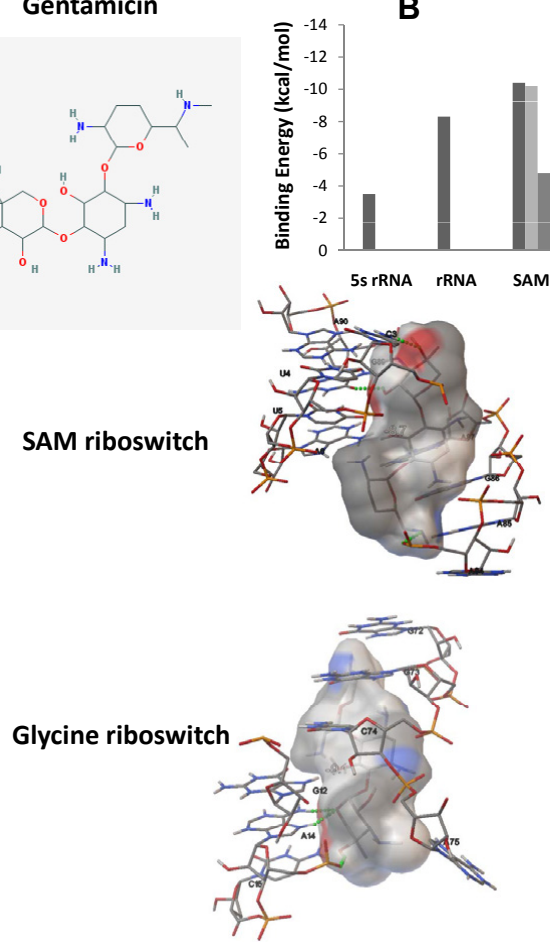

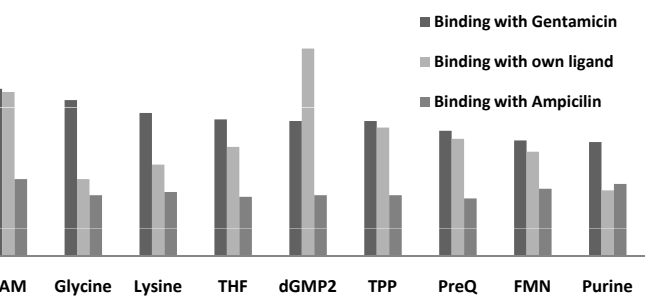

Riboswitches

A site

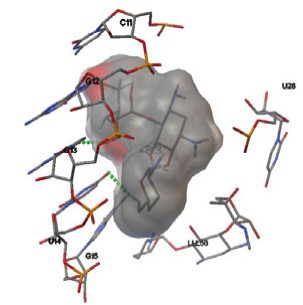

Lysine riboswitch

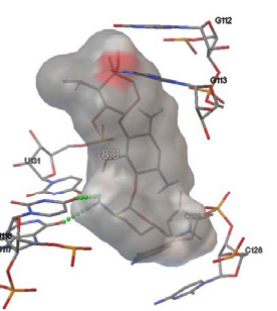

Figure 2: (A) Chemical structure of gentamicin. (B) Dark black columns demonstrate the binding energies of gentamicin with different riboswitches, dark grey columns show the binding energy of each riboswitch with its own natural ligands and light grey columns show the binding energy of each riboswitch with ampicillin based on Auto Dock Vina results. (C) The interaction between the riboswitches and gentamicin. Hydrogen bindings are shown as green dot lines.
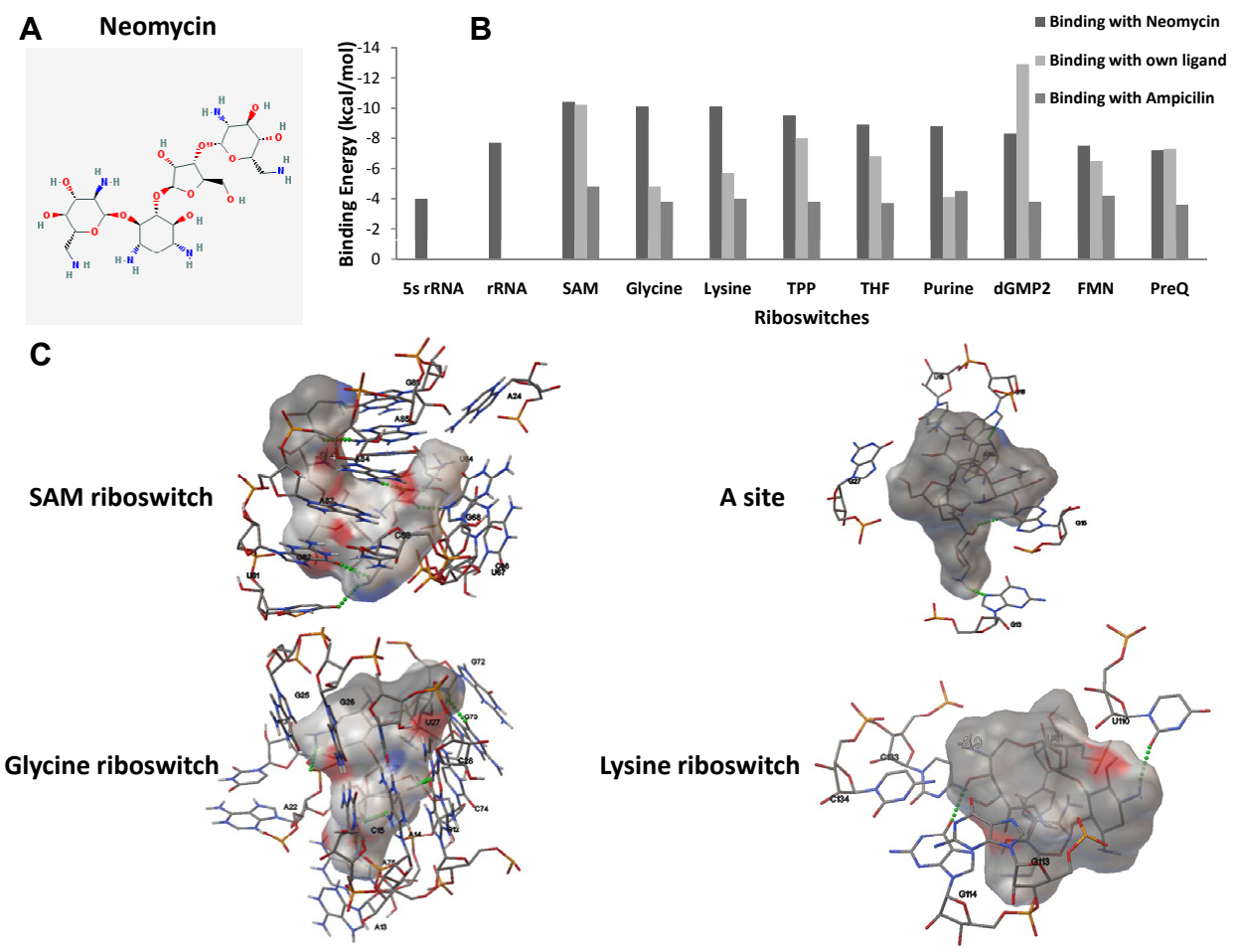

Figure 3: (A) Chemical structure of neomycin. (B) Dark black columns show the binding energies of neomycin with different riboswitches, dark grey columns show the binding energy of each riboswitch with its own natural ligands and light grey columns show the binding energy of each riboswitch with ampicillin based on Auto Dock Vina results. (C) The interaction between the riboswitches and neomycin. Hydrogen bindings are shown as green dot lines. 
$\mathbf{A}$

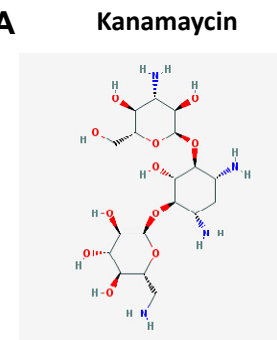

C

SAM riboswitch

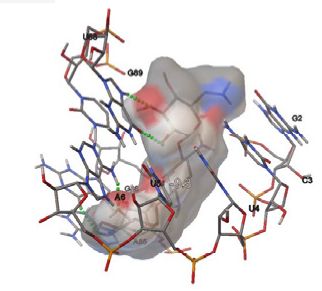

Glycine riboswitch

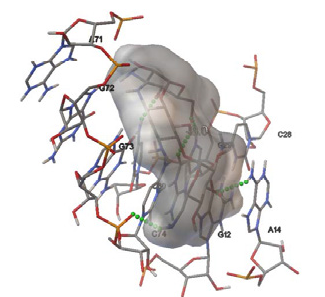

B

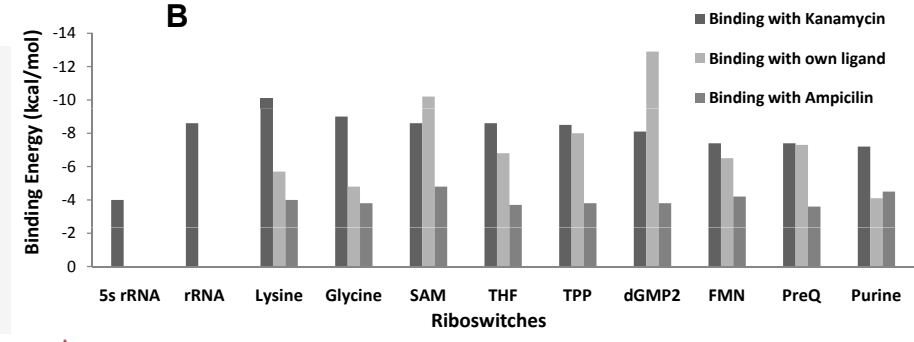

Lysine riboswitch

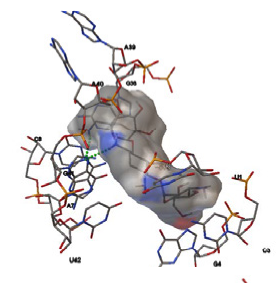

A site

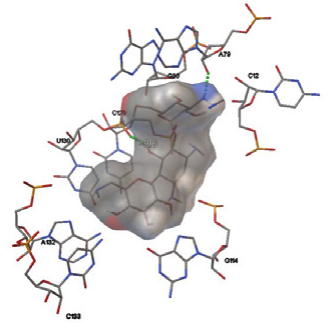

Figure 4: (A) Chemical structure of kanamycin. (B) Dark black columns represent the binding energies of kanamycin with different riboswitches, dark grey columns show the binding energy of each riboswitch with its own natural ligands and light grey columns show the binding energy of each riboswitch with ampicillin based on AutoDock Vina results. (C) The interaction between the riboswitches and kanamycin. Hydrogen bindings are demonstrated with green dot lines.

A

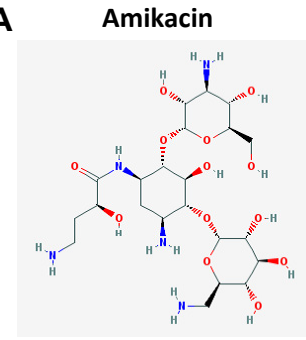

C
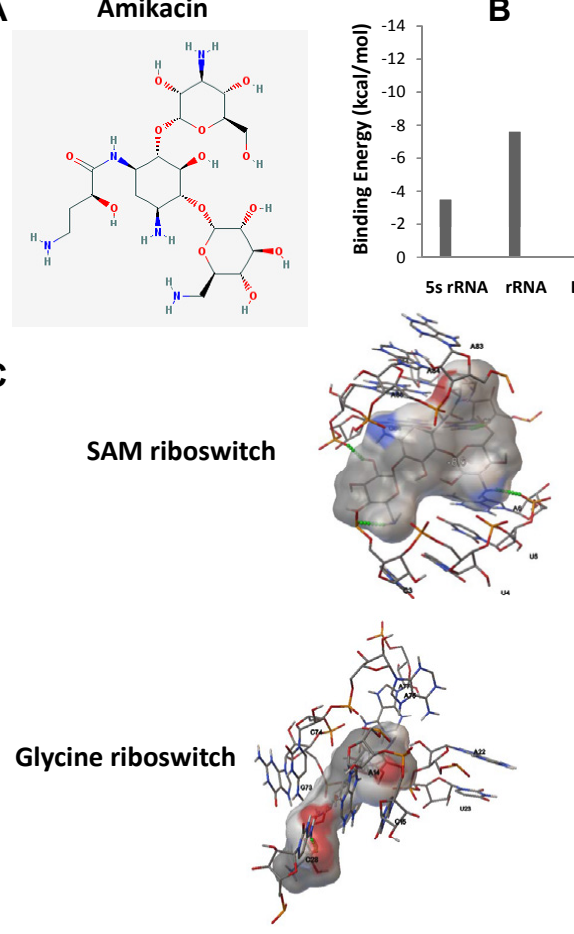

B

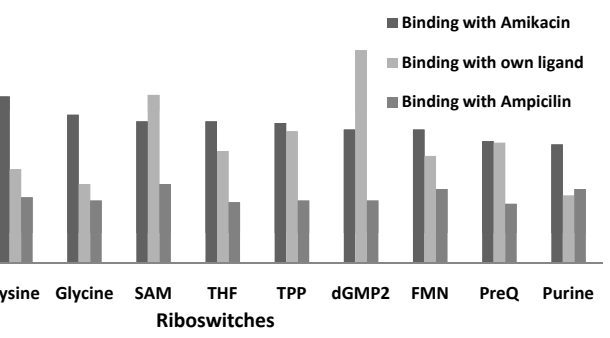

A site

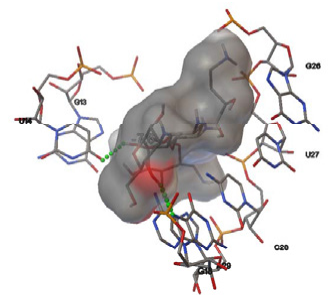

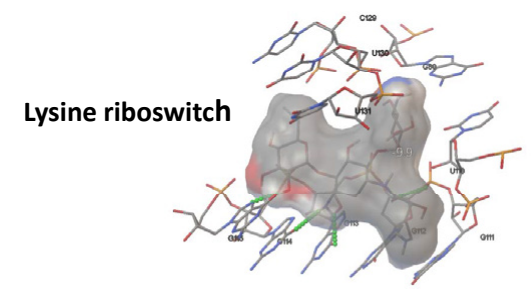

Figure 5: (A) Chemical structure of amikacin. (B) Dark black columns demonstrate the binding energies of amikacin with different riboswitches, dark grey columns show the binding energy of each riboswitch with its own natural ligands and light grey columns show the binding energy of each riboswitch with ampicillin based on AutoDock Vina results. (C) The interaction between the riboswitches and amikacin. Hydrogen bindings are shown as green dot lines. 

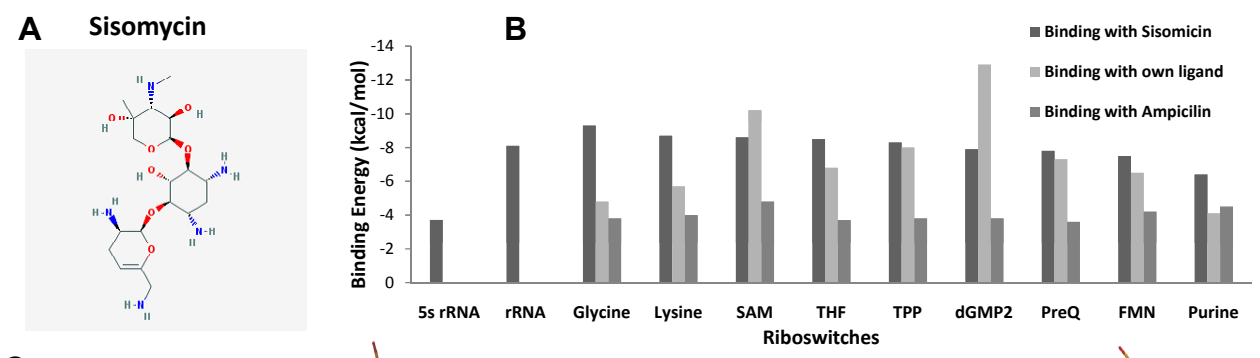

C

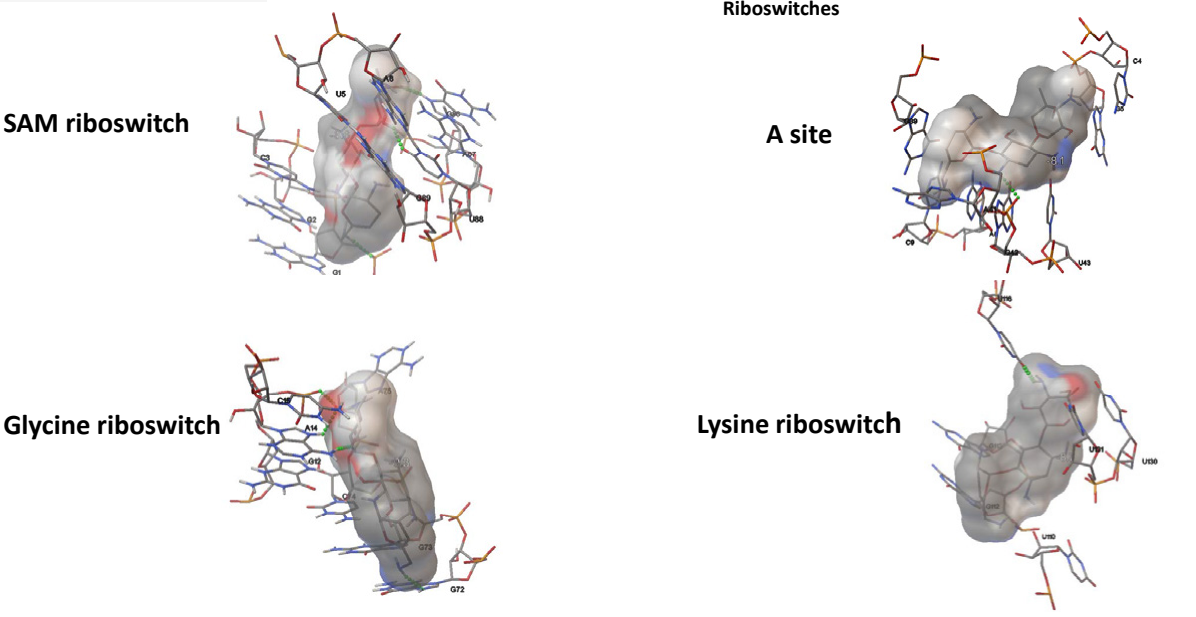

Figure 6: (A) Chemical structure of sisomicin. (B) Dark black columns show the binding energies of sisomicin with different riboswitches, dark grey columns show the binding energy of each riboswitch with its own natural ligands and light grey columns show the binding energy of each riboswitch with ampicillin based on AutoDock Vina results. (C) The interaction between the riboswitches and sisomicin. Hydrogen bindings are shown as green dot lines.

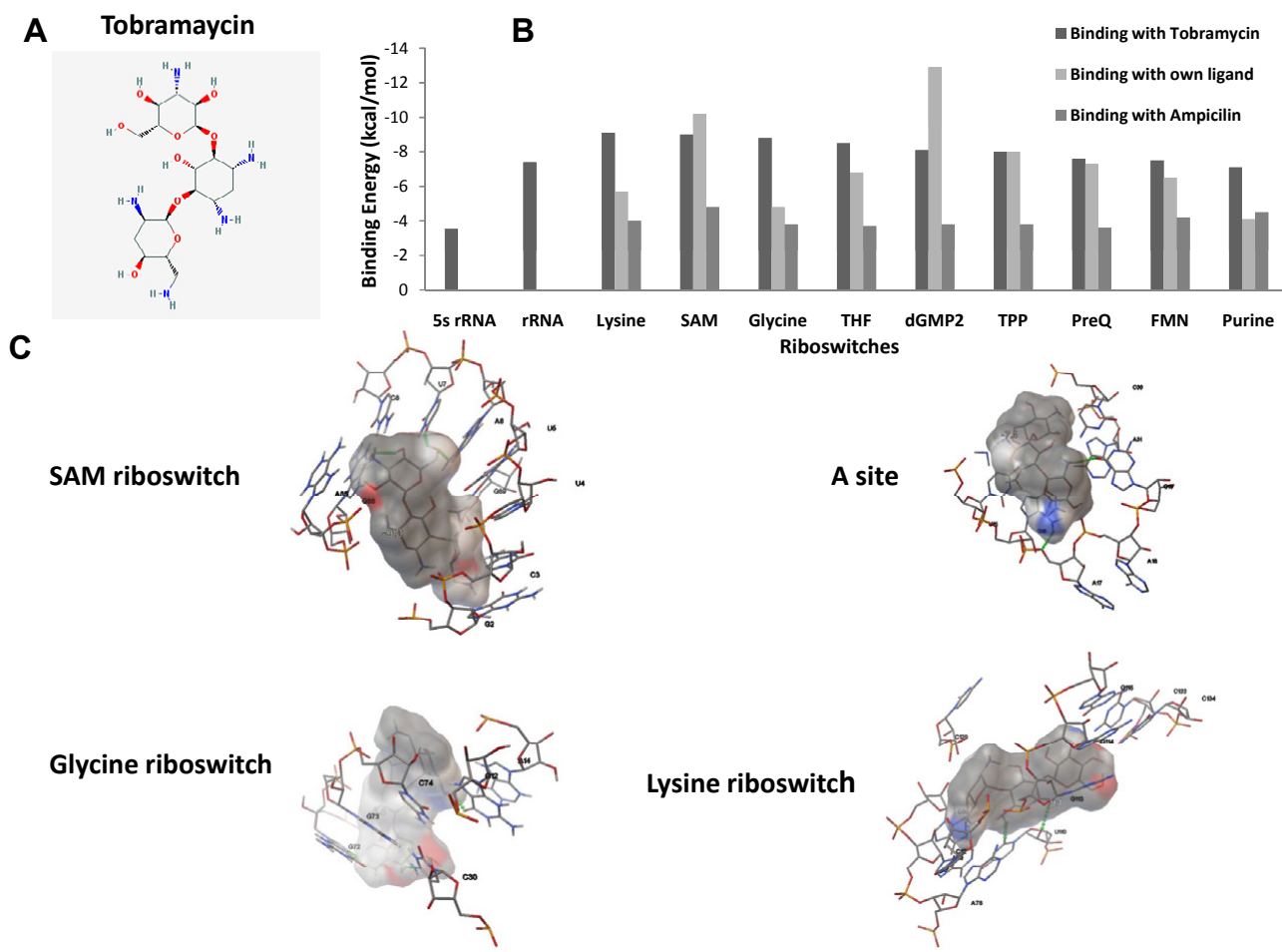

Figure 7: (A)Chemical structure of tobramycin. (B) Dark black columns demonstrate the binding energies of tobramycin with different riboswitches, dark grey columns show the binding energy of each riboswitch with its own natural ligands and light grey columns show the binding energy of each riboswitch with ampicillin based on AutoDock Vina results. (C) The interaction between the riboswitches and tobramycin. Hydrogen bindings are shown as green dot lines. 
A

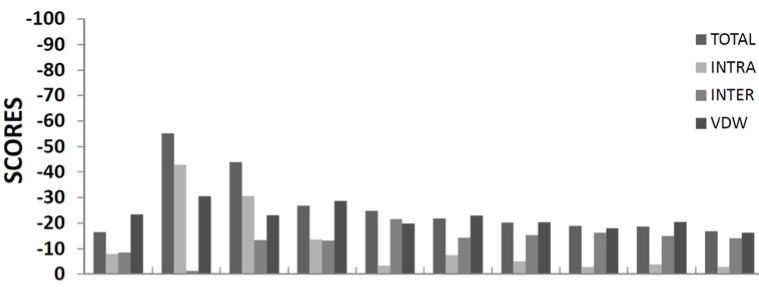

rRNA Lysine THF SAM Purine Glycine TPP preQ FMN dGMPI

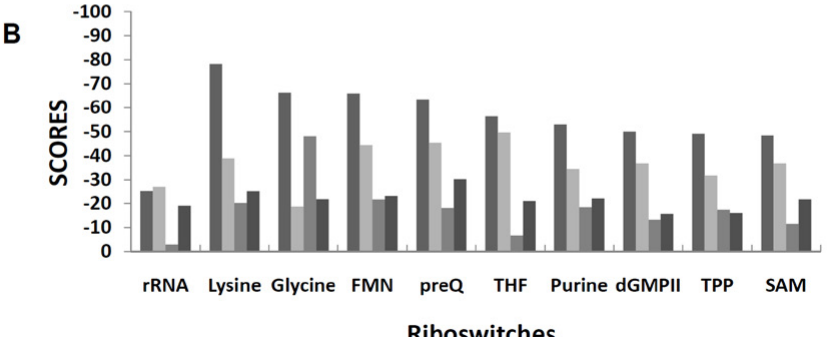

Figure 8: (A) Total, intra, inter and van der waals scores of docking paromomycin against different riboswitches acquired by rDock program.(B) Total, intra, inter and van der waals scores of docking gentamicin against different riboswitches acquired by rDock program.
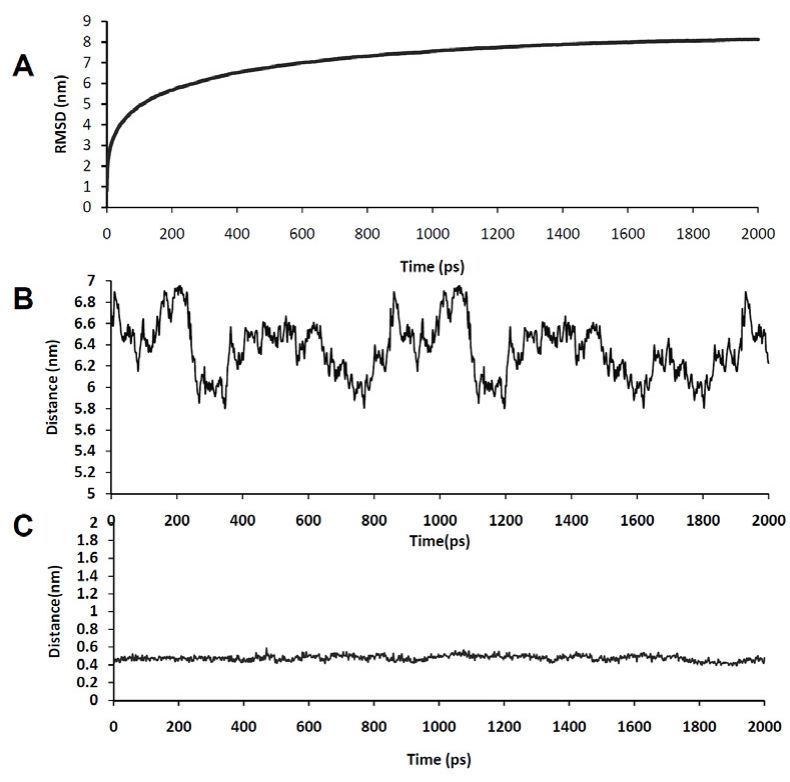

Figure 9: (A) Time dependences of RMSDs (nm) of system relative to the initial system in MD simulation. (B) Time courses of length between C4 of paromomycin and $\mathrm{P}$ of $\mathrm{A} 138$ in lysine riboswitch in negative control and (C) docked structure during MD simulation.

according to Figure 1B, lysine, glycine and SAM-I riboswitches have the best binding energies to paromomycin in comparison to " $16 \mathrm{~S}$ rRNA A site". Figure 1C illustrates the 3D structure of the interactions between paromomycin and lysine, glycine and SAM-I riboswitches and also "16S rRNA A site". The green dot lines illustrate the hydrogen binding between the ligand and the RNA molecule. It was shown that hydrogen bindings exist mostly between paromomycin and guanine and adenine nucleotides.

Approximately the same pattern was observed for other types of aminoglycosides (Figures 2-7). As seen, of 9 classes of riboswitches, lysine, glycine and SAM-I riboswitches showed higher binding energy to interact with different aminoglycosides. For some riboswitches, the natural metabolites binding energies are less than aminoglycosides' binding energies and vice versa for others. For instance, c-di-GMPII riboswitch showed higher affinity to its own metabolite ligand in comparison to the binding energies of paromomycin (Figure 1B), gentamicin (Figure 2B) and neomycin (Figure 3B). However, for kanamycin (Figure 4B), amikacin (Figure 5B), sisomicin (Figure 6B) and tobramycin (Figure 7B), in addition to c-di-GMP-II riboswitch, SAM-I riboswitch also showed higher binding energy with their own ligands. Regarding interaction between antibiotics with lysine, glycine, SAM-I riboswitches and "16s rRNA A site", most hydrogen bindings make connection between the ligands and guanine/adenine nucleotides.

\section{Docking validation}

rDock program which can be used for docking against nucleic acids very efficiently, was applied to rescore docked conformations. The conformations with higher affinity were conducted as ligands to be docked once again with rDock program. The main jobs were performed by rbcavity (cavity generation) and rbdock (docking programs) [33].

As shown in Figure 8A, according to total scores, all of the studied riboswitches showed considerable affinity to paromomycin in the range of -16.83--55.16 for c-d-GMP-II and lysine riboswitches, respectively. Then, the best total score belongs to lysine and THF riboswitches with the total score of -55.16 and -43.93 . Regarding total score of -16.49 for "A site rRNA", it can be inferred that all studied riboswitches showed more affinity to paromomycin than its known target. Also, according to Figure $8 \mathrm{~B}$, gentamicin showed even higher affinity to different riboswitches in the range of $-48.39--78.32$ for lysine and SAM-I riboswitches, respectively. Then, lysine riboswitch is also the best target for gentamicin in comparison to other riboswitches. Moreover, the difference between the total scores of riboswitches and "A site rRNA" (-25.33) is more than the case of paromomycin. In addition, the intermolecular scores are higher than intramolecular scores in paromomycin. However, in the case of gentamicin intermolecular scores are lower which reflects some differences in the involved forces of binding. On the other hand, van der waals forces are approximately equal between two aminoglycosides/riboswitches bindings.

\section{Molecular dynamics simulation}

GROMACS 5.0 suite program was used to confirm the docking results and evaluate the interaction in an environment filled with water, $\mathrm{Mg}^{2+}$ and $\mathrm{Cl}^{-}$ions. The PDB structure of docked lysine riboswitch and paromomycin was applied as the starting structure. In addition, separate ligand and riboswitch structure (with approximate distance of $6 \AA$ ) was simulated as negative control.

Figure 9A demonstrates the Root-Mean-Square Deviation (RMSD) of the whole system relative to the initial system during the simulation time. As shown, the system reaches equilibrium after 1500 ps and RMSD value of $8 \mathrm{~nm}$.

Figure 9B and Figure 9C shows the distances fluctuation between $\mathrm{C} 4$ atom of paromomycin and $\mathrm{P}$ atom of A138 in lysine riboswitch. Results showed that, the distances fluctuation is very high in negative control (Figure 9B), whereas the distances variation is very low in docked form of complex (Figure 9C). In other words, the interaction between the aminoglycoside and the riboswitch is strong enough to keep the ligand in the complex form during the simulation. 


\section{Discussion}

Non-coding RNAs have been considered as important elements for gene regulations in the cell more than a decade ago. A sort of cis acting riboregulators, so-called riboswitches, has attracted so many attentions in this regard since their discovery in 2002 [2]. Their structures consist of two essential parts including aptamer domain and expression platform. Structurally conserved aptamer domain binds to specific ligand and makes alteration in the dimensional structures of expression platform allosterically, followed by down-regulation or up-regulation of the corresponding genes [38]. Several types of riboswitches with specificity to particular ligands have been identified up to now [1]. However, lots are looking forwards to be discovered. The possibility of antibiotic targeting by riboswitches has been studied considerably. Lysine [6] and guanine [39] riboswitches are most studied targets for antibiotic designing. Although, the success to design completely effective antibiotics is rare [40], full efforts have been taken to improve drug discovery in this regard.

Aminoglycosides are important tools for battling severe infections. Their known mechanism is related to halting the translation through binding to $16 \mathrm{~S}$ rRNA A site structure [7]. In addition, the binding of some aminoglycosides to artificial riboswitches has been investigated in the number of studies [20-23]. On the other hand, a comprehensive study on the similarity of rRNA and different riboswitches structures has been conducted and possible targeting of riboswitches by paromomycin has been suggested based on docking results [27]. Accordingly in this study, the affinity of other aminoglycosides was investigated and verified via AutoDock Vina and rDock programs, respectively. Moreover, a sample MD simulation was conducted to evaluate the interaction in water and ions environment. In this study, first the PDB structures of the representatives of nine kinds of riboswitches were extracted. It should be noted that based on different studies on bound and free-state of the riboswitches, the RMSD of the atoms are not high (approximately lower than $2 \mathrm{~A}^{\circ}$ ) [41]. It means that the cell environment itself could fold the riboswitches properly [42]. Therefore, in spite of the rare PDB codes for free-state riboswitches, docking on their bound structure could be acceptable as well. However, selecting the unbound state was preferable. As shown in Figures 1-7, the binding energies of different riboswitches with related aminoglycosides are approximately similar or more than the affinity of the 16S rRNA A site with the aminoglycoside. However the binding energy of the lysine, glycine and SAM-I riboswitches are significantly higher than those of other riboswitches. In the case of paromomycin, lysine, THF and SAM-I riboswitches were best receptors according to our previous study [27]. This could be due to differences in the scoring function of AutoDock 4 (mostly based on electrostatic forces) and AutoDock Vina (mostly based on hydrophobic and hydrogen binding). However, lysine and SAM-I riboswitches have been considered as better targets according to both methods. In addition, the affinity of riboswitches/ aminoglycosides is more than the binding energy of negative controls including riboswitches/ampicillin and $5 \mathrm{~S}$ rRNA/aminoglycosides complexes (Figure 1-7). The exception of c-di-GMP-II riboswitch for all aminoglycosides and SAM-I riboswitch for kanamycin (Figure 4B), amikacin (Figure 5B), sisomicin (Figure 6B) and tobramycin (Figure 7B) demonstrates that these riboswitches may fail to bind aminoglycosides in competition with their known ligands. Binding characteristics of different aminoglycosides showed that strong hydrogen bindings exist between the aminoglycoside molecules and guanine/adenine nucleotides. In this regard, there is no difference between the binding of aminoglycoside/A site and aminoglycoside/riboswitch interactions.
rDock program as a valid and strong newly developed docking method for ribonucleic acids [33] have been utilized to rescore the results of AutoDock Vina. Based on the observations (Figure 8) almost all riboswitches/aminoglycoside demonstrate higher total scores in comparison to $\mathrm{rRNA} / \mathrm{aminoglycosides}$ in the case of paromomycin and gentamicin. Showing high total score, lysine riboswitch can be considered as the most important target for aminoglycosides which is in accordance with the results of Auto Dock Vina and Autodock 4 [27]. However, all riboswitches illustrated better affinity to aminoglycosides in comparison to rRNAs. According to the results, although the binding modes of paromomycin and gentamicin may be different with each other, vdw forces are almost equal among riboswitches and aminoglycosides.

Taken the whole, it seems that though different riboswitches show affinity to aminoglycosides, some of them are particularly better than others in terms of higher binding energy such as lysine riboswitch verified by rDock scoring function.

It should be noted that molecular docking has a number of limitations beside its strength. For example, its dependence on the search algorithm and scoring function makes the performance of this approach quite different. Besides, the rigidity of binding site is not quite similar with what happens in reality. In addition, calculation of the binding energy in the vacuum condition without considering the effect of environmental molecules is an important limitation of docking method. Particularly, the presence of cationic ions such as $\mathrm{Mg}^{2+}$ is so important for the behavior of the riboswitches in the cell. As a result, in the next step, the complex of lysine riboswitch/ paromomycin was studied through MD simulation. MD simulation enabled us to evaluate the interaction in the mixture of water and ions, especially $\mathrm{Mg}^{2+}$. Regarding the RMSD value the system is equilibrated. The result of the simulation showed that the distance of the ligand and the receptor was not varied considerably during the simulation, while the distance variation was considerably high in the negative control simulation (Figure 9). These findings approved the possibility of interaction between lysine riboswitch and paromomycin even in the ionic environment.

However, computational methods cannot replace experimental validation of the results and further in vitro and in vivo studies are needed to confirm the results.

\section{Conclusion}

In this study the binding affinity of 7 aminoglycosides including paromomycin, gentamicin, amikacin, kanamycin, neomycin, tobramaycin and sisomicin to different types of riboswitches was evaluated and validated though docking methods and MD simulation. In conclusion, according to Auto Dock Vina results, almost all kinds of aminoglycosides have considerable affinities to the riboswitches. Among them lysine, glycine and SAM-I riboswitches showed higher binding energies. In addition, the binding energies of riboswitches/ aminoglycosides are more than the binding energies of negative control complexes including riboswitches/ampicillin and 5S rRNA/ aminoglycosides. Additionally, most hydrogen bindings are created with guanine and adenine nucleotides. Docking validation through rDock program confirmed data as it was shown that the total score is higher in all riboswitches/aminoglycosides interactions in comparison with "16S rRNA A site"/aminoglycosides interactions. MD simulation study on lysine riboswitch/paromomycin complex approved the docking results even within the solvent in the presence of magnesium and chloride ions.. Further computational and experimental studies are 
Citation: Mehdizadeh Aghdam E, Esmaeil Hejazi M, Hejazi MS, Barzegar A (2014) Riboswitches as Potential Targets for Aminoglycosides Compared with rRNA Molecules: In Silico Study. J Microb Biochem Technol S9: 002. doi:10.4172/1948-5948.S9-002

suggested regarding interaction of riboswitches with aminoglycosides as well as consequences, effectiveness and efficiency of the interaction.

\section{Acknowledgment}

This article was written based on a data set of Ph.D thesis of Elnaz Mehdizadeh Aghdam registered in Tabriz University of Medical Sciences. This study has been supported financially under Ph.D thesis proposal submitted at No. 80 in the Faculty of Pharmacy, Tabriz University of Medical Sciences, Tabriz, Iran.

\section{References}

1. Winkler WC, Breaker RR (2005) Regulation of bacterial gene expression by riboswitches. Annu Rev Microbiol 59: 487-517.

2. Nahvi A, Sudarsan N, Ebert MS, Zou X, Brown KL, et al. (2002) Genetic control by a metabolite binding mRNA. Chem Biol 9: 1043.

3. Serganov A, Huang L, Patel DJ (2009) Coenzyme recognition and gene regulation by a flavin mononucleotide riboswitch. Nature 458: 233-237.

4. Lee ER, Blount KF, Breaker RR (2009) Roseoflavin is a natural antibacteria compound that binds to $\mathrm{FMN}$ riboswitches and regulates gene expression. RNA Biol 6: 187-194

5. Sudarsan N, Cohen-Chalamish S, Nakamura S, Emilsson GM, Breaker RR (2005) Thiamine pyrophosphate riboswitches are targets for the antimicrobia compound pyrithiamine. Chem Biol 12: 1325-1335.

6. Blount KF, Wang JX, Lim J, Sudarsan N, Breaker RR (2007) Antibacterial lysine analogs that target lysine riboswitches. Nat Chem Biol 3: 44-49.

7. Carter AP, Clemons WM, Brodersen DE, Morgan-Warren RJ, Wimberly BT, et al. (2000) Functional insights from the structure of the $30 \mathrm{~S}$ ribosomal subunit and its interactions with antibiotics. Nature 407: 340-348.

8. Fourmy D, Recht MI, Blanchard SC, Puglisi JD (1996) Structure of the A site of Escherichia coli 16S ribosomal RNA complexed with an aminoglycoside antibiotic. Science 274: 1367-1371.

9. Davies J, Davis BD (1968) Misreading of ribonucleic acid code words induced by aminoglycoside antibiotics. The effect of drug concentration. J Biol Chem 243: 3312-3316

10. Hermann T, Westhof E (1999) Docking of cationic antibiotics to negatively charged pockets in RNA folds. J Med Chem 42: 1250-1261.

11. Faber $C$, Sticht $H$, Schweimer K, Rösch $P$ (2000) Structural rearrangements of HIV-1 Tat-responsive RNA upon binding of neomycin B. J Biol Chem 275: 20660-20666.

12. Kirk SR, Luedtke NW, Tor $Y$ (2000) Neomycin-acridine conjugate: a potent inhibitor of Rev-RRE binding. J Am Chem Soc 122: 980-981.

13. Rogers J, Chang AH, von Ahsen U, Schroeder R, Davies J (1996) Inhibition of the self-cleavage reaction of the human hepatitis delta virus ribozyme by antibiotics. J Mol Biol 259: 916-925.

14. Mikkelsen NE, Brännvall M, Virtanen A, Kirsebom LA (1999) Inhibition of RNase P RNA cleavage by aminoglycosides. Proc Natl Acad Sci U S A 96: 6155-6160.

15. Stage TK, Hertel KJ, Uhlenbeck OC (1995) Inhibition of the hammerhead ribozyme by neomycin. RNA 1: 95-101.

16. von Ahsen U, Davies J, Schroeder R (1991) Antibiotic inhibition of group ribozyme function. Nature 353: 368-370.

17. Murchie AI, Davis B, Isel C, Afshar M, Drysdale MJ, et al. (2004) Structurebased drug design targeting an inactive RNA conformation: exploiting the flexibility of HIV-1 TAR RNA. J Mol Biol 336: 625-638.

18. Davis B, Afshar M, Varani G, Murchie Al, Karn J, et al. (2004) Rational design of inhibitors of HIV-1 TAR RNA through the stabilisation of electrostatic "hot spots". J Mol Biol 336: 343-356

19. Famulok M, Hüttenhofer A (1996) In vitro selection analysis of neomycin binding RNAs with a mutagenized pool of variants of the 16S rRNA decoding region. Biochemistry 35 : 4265-4270.

20. Weigand JE, Sanchez M, Gunnesch EB, Zeiher S, Schroeder R, et al. (2008) Screening for engineered neomycin riboswitches that control translation initiation. RNA 14:89-97.

21. Morse DP (2007) Direct selection of RNA beacon aptamers. Biochem Biophys Res Commun 359: 94-101.
22. Schmidtke SR, Duchardt-Ferner E, Weigand JE, Suess B, Wöhnert J (2010) NMR resonance assignments of an engineered neomycin-sensing riboswitch RNA bound to ribostamycin and tobramycin. Biomol NMR Assign 4: 115-118.

23. Vandenengel JE, Morse DP (2009) Mutational analysis of a signaling aptamer suggests a mechanism for ligand-triggered structure-switching. Biochem Biophys Res Commun 378: 51-56.

24. Lünse CE (2012) Investigation of riboswitches as new antibacterial targets: Identification and characterization of novel synthetic and natural riboswitch modulators with effect on bacterial cell growth [Ph.D.]. Bonn: Rheinischen Friedrich-Wilhelms-Universität Bonn. 218 p.

25. Barrick JE, Breaker RR (2007) The distributions, mechanisms, and structures of metabolite-binding riboswitches. Genome Biol 8: R239.

26. Duchardt-Ferner E, Weigand JE, Ohlenschläger O, Schmidtke SR, Suess B, et al. (2010) Highly modular structure and ligand binding by conformational capture in a minimalistic riboswitch. Angew Chem Int Ed Engl 49: 6216-6219.

27. Mehdizadeh Aghdam E, Barzegar A, Hejazi MS (2014) Evolutionary Origin and Conserved Structural Building Blocks of Riboswitches and Ribosomal RNAs: Riboswitches as Probable Target Sites for Aminoglycosides Interaction. Adv Pharm Bull 4: 225-235

28. Barzegar A, Moosavi-Movahedi AA, Mahnam K, Ashtiani SH (2010) Chaperonelike activity of alpha-cyclodextrin via hydrophobic nanocavity to protect native structure of ADH. Carbohydr Res 345: 243-249.

29. Morris GM, Lim-Wilby M (2008) Molecular docking. Methods Mol Biol 443: 365 382

30. Morris GM, Huey R, Lindstrom W, Sanner MF, Belew RK, et al. (2009) AutoDock4 and AutoDockTools4: Automated docking with selective receptor flexibility. J Comput Chem 30: 2785-2791.

31. Trott O, Olson AJ (2010) AutoDock Vina: improving the speed and accuracy of docking with a new scoring function, efficient optimization, and multithreading J Comput Chem 31: 455-461.

32. Breaker RR (2012) Riboswitches and the RNA world. Cold Spring Harb Perspect Biol 4.

33. Ruiz-Carmona S, Alvarez-Garcia D1, Foloppe N2, Garmendia-Doval AB3 Juhos S4, et al. (2014) rDock: a fast, versatile and open source program for docking ligands to proteins and nucleic acids. PLoS Comput Biol 10: e1003571.

34. Pronk S, Páll S, Schulz R, Larsson P, Bjelkmar P, et al. (2013) GROMACS 4.5 a high-throughput and highly parallel open source molecular simulation toolkit. Bioinformatics 29: 845-854.

35. Jorgensen WL, Chandrasekhar J, Madura JD, Impey RW, Klein ML (1983) Comparison of simple potential functions for simulating liquid water. $\mathrm{J}$ Chem Phys 79: 926-935.

36. Bjelkmar Pr, Larsson P, Cuendet MA, Hess B, Lindahl E (2010) Implementation of the CHARMM force field in GROMACS: Analysis of protein stability effects from correction maps, virtual interaction sites, and water models. J Chem Theory Comput 6: 459-466.

37. Trott O, Olson AJ (2010) AutoDock Vina: improving the speed and accuracy of docking with a new scoring function, efficient optimization, and multithreading J Comput Chem 31: 455-461.

38. Nudler E, Mironov AS (2004) The riboswitch control of bacterial metabolism Trends Biochem Sci 29: 11-17.

39. Mulhbacher J, Brouillette E, Allard M, Fortier LC, Malouin F, et al. (2010) Nove riboswitch ligand analogs as selective inhibitors of guanine-related metabolic pathways. PLoS Pathog 6: e1000865.

40. Ster C, Allard M, Boulanger S, Lamontagne Boulet M, Mulhbacher J, et al (2013) Experimental treatment of Staphylococcus aureus bovine intramammary infection using a guanine riboswitch ligand analog. J Dairy Sci 96: 1000-1008.

41. Liberman JA, Wedekind JE (2012) Riboswitch structure in the ligand-free state Wiley Interdiscip Rev RNA 3: 369-384.

42. Tyrrell J, McGinnis JL, Weeks KM, Pielak GJ (2013) The cellular environment stabilizes adenine riboswitch RNA structure. Biochemistry 52: 8777-8785. 